\title{
Alimentação saudável na infância: um relato de experiência
}

\author{
Healthy eating in childhood: report of experience
}

Alimentación saludable en la infancia: un relato de experiencia

Roselane Ferreira Seabra ${ }^{1 *}$, Ewelly Thais Sandoval Silva ${ }^{1}$, Fernanda Larissa Matos de Lima1, Maria Clara Oliveira da Luz ${ }^{1}$, Yasmin da Silva Santos ${ }^{1}$, Laura Larissa Nogueira Pereira ${ }^{1}$, Maria Rute de Souza Araújo'.

\section{RESUMO}

Objetivo: Relatar uma ação de educação em saúde, onde foram usadas tecnologias educativas para observar e orientar mães em relação ao preparo e oferta nutricional correta a crianças de 6-12 meses em uma Unidade Básica de Saúde (UBS). Relato de experiência: Foi conduzido por seis acadêmicas de enfermagem do sexto semestre de uma universidade privada do Estado do Pará. Foi desenvolvida uma atividade com intuito de orientar as mães a respeito do preparo e oferta nutricional correta a crianças de 6-12 meses. Sendo prosseguida pela realização de uma dinâmica, fazendo com que as mães montassem pratos com os alimentos disponibilizados pelos facilitadores da ação, de acordo com seus conhecimentos a respeito da alimentação para crianças de 6-12 meses. Considerações finais: A ação educativa permitiu identificar a inserção precoce de alimentos, como: ultraprocessados, ingestão precoce de sal e açúcar, leites artificiais e alimentos com baixo teor nutricional. Além de questões biopsicossocioculturais, que também estão relacionadas com o tipo de ingesta alimentar. A ação possibilitou informar as mães acerca de como iniciar de forma saudável e gradual, a introdução de alimentos nutritivos para a crianças ainda na primeira infância, visando sempre a condição socioeconômica da família.

Palavras-chave: Alimentação saudável, Criança, Educação em saúde.

\begin{abstract}
Objective: To report a health education action, where educational technologies were used to observe and guide mothers in relation to the correct nutritional preparation and offer to children aged 6-12 months in a Basic Baúde Unit (UBS). Experience report: It was conducted by six nursing students from the sixth semester of a private university in the State of Pará. An activity was developed in order to guide mothers about the correct nutritional preparation and offer to children aged 6-12 months. It was continued by carrying out a dynamic, making the mothers assemble plates with the food provided by the action facilitators, according to their knowledge about food for children aged 6-12 months. Final considerations: The educational action allowed to identify the early inclusion of foods, such as: ultra-processed foods, early intake of salt and sugar, artificial milk and foods with low nutritional content. In addition to biopsychosociocultural issues, which are also related to the type of food intake. The action made it possible to inform mothers about how to start, in a healthy and gradual way, the introduction of nutritious foods for children in early childhood, always aiming at the socioeconomic condition of the family.
\end{abstract}

Keywords: Healthy eating, Children, Health education.

\section{RESUMEN}

Objetivo: informe de una acción de educación en salud, donde se utilizaron tecnologías para observar y orientar a las madres en relación a la correcta preparación y oferta nutricional para los niños de 6 a 12 meses en una Unidad Básica Saúde (UBS). Informe de experiencia: Fue realizado por seis estudiantes de enfermería del sexto semestre de una universidad privada del Estado de Pará, se desarrolló una actividad

${ }^{1}$ Centro Universitário Metropolitano da Amazônia (UNIFAMAZ), Belém - PA.

*E-mail: roselaneseabra@gmail.com 
para orientar a las madres sobre la correcta preparación y oferta nutricional a los niños de 6 a 12 meses. Se continuó con la realización de una dinámica, haciendo que las madres ensamblen platos con la comida que les brindan los facilitadores de la acción, de acuerdo con sus conocimientos sobre alimentación para niños de 6 a 12 meses. Consideraciones finales: La acción educativa debe identificar la inclusión temprana de alimentos, tales como: alimentos ultraprocesados, ingesta temprana de sal y azúcar, leches artificiales y alimentos de bajo contenido nutricional. Además de las cuestiones biopsicosocioculturales, que también están relacionadas con el tipo de ingesta alimentaria. La acción permitió informar a las madres sobre cómo iniciar, de manera saludable y paulatina, la introducción de alimentos nutritivos para los niños en la primera infancia, formando siempre la condición socioeconómica de la familia.

Palabras clave: Alimentación saludable, Niños, Educación para la salud.

\section{INTRODUÇÃO}

Os primeiros anos de vida da criança são acompanhados de diversas mudanças e avanços. Sendo assim, é uma fase a qual o cuidado nutricional faz-se importante para que esses progressos ocorram de forma satisfatória e, principalmente, para que o organismo da criança consiga estar apto a se defender contra diversas patologias que possam acometê-la durante o período de maturação das suas estruturas fisiológicas (SILVA IE, et al., 2020).

Sendo assim, o conhecimento acerca da oferta nutricional apropriada à criança demanda atenção para que haja mudanças e melhoras em relação aos cuidados alimentares voltados à esta população. Onde, por meio do papel de educador, o enfermeiro age incentivando a conscientização voltada para a temática nutricional a fim de promover a educação continuada. Percebe-se então, os profissionais de enfermagem como agentes importantes do processo de orientar tais cuidados, por estarem à frente de diversos programas na Atenção Primária em Saúde (APS), tendo o primeiro contato com o paciente (NARCHI NZ, et al., 2019).

A enfermagem atua no sentido de promover informações que incentivam condutas apropriadas. Como é o caso do aleitamento materno, que é estimulado desde o pré-natal e é de grande importância para o lactente, pois o leite materno traz vários benefícios, como: proteger a criança de doenças alérgicas, desnutrição, doenças digestivas, obesidade, cáries, reduz a morbidade nas crianças prematuras, além de auxiliar na maturação do sistema gastrointestinal, desenvolvimento psicomotor e resultar em benefícios para o sistema imunológico (SILVA IE, et al., 2020).

As normas da Atenção Integradas às Doenças Prevalentes na Infância (AIDPI) apontam os hábitos alimentares inadequados nos primeiros anos da infância como fatores de risco importantes para o surgimento de doenças prevalentes. Desse modo, é evidenciada a importância do papel de educador atribuído aos profissionais de enfermagem para orientar os genitores dentro da APS acerca de informações relacionadas à nutrição ideal para cada fase da vida da criança desde a importância do leite materno até a incentivar a criança a comer de forma independente (MINISTÉRIO DA SAÚDE, 2017a).

A partir dos 6 meses, além do leite materno, novos alimentos são introduzidos na alimentação da criança e nesse momento é apresentando um novo universo de cores, sabores, texturas e cheiros a criança (BRASIL, 2018). A variedade nutricional cresce ao passo em que o bebê atinge outras faixas etárias. Portanto, é preciso garantir que a ingesta alimentar seja adequada para satisfazer suas necessidades fisiológicas, fazendo-se necessário introduzir alimentos com maior teor nutricional (CARLETTI CO, et al., 2019).

As tecnologias educacionais são utilizadas por profissionais de saúde com o objetivo de disseminar conhecimentos acerca de assuntos que necessitam de um olhar assertivo. Logo, as tecnologias educacionais atuam no sentido de dar autonomia ao paciente, agindo como mecanismo de transformação através da instrução de novas práticas (SILVEIRA RSO, et al., 2016).

A consulta de enfermagem na puericultura, tem papel fundamental na concepção de vínculos e na oferta de uma atenção humanizada. O enfermeiro utiliza como instrumento organizacional de cuidados a Sistematização da Assistência de Enfermagem (SAE) instituída pela resolução 358/2009 do Conselho Federal 
de Enfermagem (COFEN) que dispõe sobre a SAE e a implementação do Processo de Enfermagem (PE). Logo, realizar um atendimento pautado na SAE, organiza e direciona as condutas de enfermagem ampliando o olhar integral e pensamento científico. Assim, durante a puericultura, o enfermeiro a utiliza como meio de implementar educação em saúde (HANZEN IP, et al., 2019).

Dessa forma, o objetivo do estudo é relatar uma ação de educação em saúde, onde foi usada uma tecnologia educativa para observar e orientar as mães, a respeito do preparo e oferta nutricional correta das crianças de 6-12 meses em uma Unidade Básica de Saúde (UBS).

\section{RELATO DE EXPERIÊNCIA}

Trata-se de uma ação de educação em saúde tipo relato de experiência, sobre alimentação saudável na infância, realizada em uma UBS de uma cidade do estado do Pará, a atividade educativa foi desenvolvida no dia 3 de novembro de 2021, às 13:00 horas, tendo como público-alvo as mães na referida instituição atendidas na UBS.

A ação de educação em saúde contou com a presença de 6 mães, as quais estavam com seus filhos presentes no momento da apresentação. A atividade foi desenvolvida com intuito de orientar a respeito do preparo e oferta nutricional correta a crianças de 6-12 meses, a ação educativa ocorreu em três momentos: No primeiro momento, foi disponibilizado para o público alvo: frutas, verduras, legumes, sal, açúcar e alimentos industrializados, sendo prosseguida pela realização de uma dinâmica, fazendo com que os presentes montassem dois pratos contendo os alimentos que estes costumam ofertar durante o lanche e 0 almoço para a criança de acordo com o seu próprio conhecimento. A partir da montagem dos pratos feitos pelas mães, foi analisado que a maioria utilizava temperos industrializados nas refeições, cozinhavam os legumes a vapor e utilizavam açúcares nos sucos de frutas, junto a salgadinhos e biscoitos adocicados.

$\mathrm{Na}$ análise da montagem do almoço, observou-se que o total de presentes utilizava alimentos comuns no cotidiano alimentar da família, como: batata, cenoura, arroz, feijão e proteínas como carne e frango sempre depositados de forma misturada e amassada no prato da criança e, e em outros momentos, eram ofertados papas artificiais feitas de aditivos conservantes.

$\mathrm{Na}$ observação da construção do lanche, as mães afirmaram introduzir iogurtes, leites artificiais, bolacha à base de sal e biscoitos sem recheio. Acerca da ingesta hídrica, os participantes relataram ser por demanda espontânea, sem valores certos para basear a ingesta hídrica diária. Essa atividade mostrou-se importante, pois naquele momento foi possível observar os conhecimentos dos pais a respeito da temática apresentada.

No segundo momento, foi conduzida uma apresentação ministrada pelos facilitadores da ação, a respeito da nutrição adequada e saudável para crianças de 6-12 meses, onde foi articulada a importância da oferta saudável e orientações pautadas em conhecimentos científicos acerca de alimentos que são comumente dados à criança e podem ser danosos à saúde durante o processo maturação gastrointestinal. Após a apresentação, houve a demonstração e análise dos itens considerados certos e errados colocados nos pratos feitos por cada mãe, gerando aos participantes reflexões e estímulo para melhoria na preparação dos alimentos, seus tipos e origens.

No terceiro momento a equipe realizou uma ação de caráter educativo, onde as mães foram orientadas sobre alimentação correta desde a primeira introdução alimentar da criança. A atividade foi desenvolvida para saber se o objetivo da ação foi alcançado, foi realizada uma dinâmica final, onde foram feitas perguntas a respeito da atividade apresentada e a distribuição de um brinde, no qual, era representado por um kit infantil formado por: um prato, um copo e uma colher infantil. O desfecho da ação culminou com a solução de dúvidas que emergiram no decorrer e no final da apresentação sobre o tema apresentado, especificamente a respeito de quais seriam os alimentos mais adequados para a primeira introdução alimentar de crianças com faixa etária de 06 meses de idade.

Ao final da ação foi doado para a unidade de saúde um banner com imagens ilustrativas e educativas, sobre alimentação saudável para crianças de 6-12 meses. 


\section{DISCUSSÃO}

A ação educativa apontou déficit no nível no conhecimento das mães em relação à composição dos alimentos, onde observou-se a inserção precoce de papas ultra processadas ingestão precoce de sal e açúcar, leites artificiais e alimentos com baixo teor nutricional. Além de questões biopsicossocioculturais, que também estão relacionadas com o tipo de ingesta alimentar. Portanto, percebe-se as questões nutricionais como uma esfera onde as sociedades constroem suas identidades baseadas em suas concepções históricas carregadas de valores culturais socialmente estruturados e influenciados por ela (RAMOS DC e COELHO TCB, 2017).

De acordo com Araújo TS, et al. (2016), os desafios encontrados durante o processo de nutrição nos primeiros anos de vida continuam sendo um problema no Brasil. Relacionando-se diretamente com as desigualdades inter-regionais e sociais que ainda apresentam-se, a diversidade dos país e a persistência de segmentos com elevada prevalência de maus hábitos nutricionais.

Sabe-se que 6 meses é um período mínimo de amamentação exclusiva, porém essa estimativa muitas vezes não é seguida e alimentos são introduzidos ainda dentro desse período, o que é um risco para obesidade infantil além de outras patologias. E mesmo quando introduzidos no momento certo, existem problemas na quantidade e formas de apresentar determinados alimentos para essas crianças (GURMINI J, et al., 2017).

Com isso, a introdução inadequada de alimentos à dieta da criança pode acabar em resultado danosos para a saúde, sobretudo quando a oferta é realizada antes do completo desenvolvimento fisiológico. A realização alimentar antes dos seis meses de vida ocasiona deficiências à saúde infantil. Com isso, muitas mães ainda acreditam que alguns líquidos podem agregar ao leite materno e trazer benefícios aos lactentes. (LOPES WC, et al., 2018).

De acordo com Morais MB, et al. (2017), crianças que foram amamentadas apresentaram escores de desenvolvimento psicomotor, mental e de linguagem superiores aos daquelas alimentadas com fórmulas infantis à base de leite de vaca ou seu derivado. Contribuindo com a ideia de Silva IE, et al. (2020), a realização alimentar da criança é caracterizada pelo leite materno e introdução de alimentos na idade correta, porém pode sofrer interferências de familiares, nesse caso a mãe deve manter o controle nos cuidados com a criança. A amamentação materna exclusiva é influenciada por diversos fatores como condições socioeconômicas, escolaridade, situação conjugal, laboral e obstétrica, idade materna e amamentação anterior, após o período de seis meses de vida a criança deverá receber alimentos pastosos e sólidos afim de complementar sua alimentação que não será mais somente o leite materno (CARDOSO AVP, et al., 2019).

E de acordo com Lopes WC, et al. (2018), da mesma forma que a introdução precoce é prejudicial, a inclusão de novos alimentos tardiamente também não é recomendada porque a partir desse momento o leite materno não mais atende às necessidades energéticas da criança

Segundo o Ministério de Saúde (2018b) alimentos complementares são ricos em energia e nutrientes e devem respeitar a identidade cultural e alimentar das diversas regiões, resgatando e valorizando os alimentos regionais; ou seja, as frutas, legumes e verduras cultivadas localmente. Deve-se buscar usar alimentos de safra e acessíveis ao nível socioeconômico familiar. Dessa forma, são atributos da alimentação complementar saudável: o acesso físico e financeiro, os sabores, as variedades, as cores, a segurança sanitária e a riqueza em nutrientes

Todos os anos a população infantil em sobrepeso aumenta devido à falta de conhecimento do responsável quanto à quantidade e qualidade dos alimentos que devem ser oferecidos, também influenciam as questões culturais, problemas financeiros nas famílias, aumento da ingestão de fast foods e ultra processados devido as grandes jornadas de trabalho e consequentemente falta de tempo do adulto para fazer o preparo da alimentação da criança (MINISTÉRIO DA SAÚDE, 2019c).

A falta de treinamento da equipe profissional de saúde também implica em um dos obstáculos que causam deficiência na vigilância do crescimento na rede básica de saúde no Brasil, a totalidade da equipe de saúde em composição mínima gera para a criança maior apoio nutricional (PEDRAZA DF e OLIVEIRA MM, 2021). 
Desta maneira, muitas mães desejam incrementar a alimentação da criança, baseadas em seus costumes ou conforme o conhecimento empírico, definindo deste modo o que é apropriado e o que é inadequado. Os hábitos alimentares também são alinhados conforme as condições socioeconômicas, onde as práticas alimentares serão refletidas na posição social, na qual serão atribuídos os alimentos com maior relevância para a família. Logo, o comportamento alimentar infantil é caracterizado e determinado no âmbito em que se encontra, pelas interações culturais, socioeconômicas e psicológicas. Recentes estudos frisam que a supervalorização do papel da família no ganho de peso ressaltada a crença de que uma criança só é saudável e bem cuidada se estiver aparentemente corpulento, isso acarreta uma falsa percepção de saúde, onde a mãe acaba não revelando a ideia futura de seu filho em relação ao sobrepeso, obesidade e futuras doenças crônicas cardiovasculares (RAMOS DC e COELHO TCB, 2017).

Ressalta-se a necessidade de uma orientação eficaz aos pais quanto a alimentação saudável da criança de 6 a 12 meses, pois foi observado uma enorme carência de informações por parte das mães quanto a montagem dos pratos e a quantidade que deve ser ofertada. Essas informações devem ser repassadas por meio de ações educativas voltadas para as mães com a finalidade de incentivar a introdução alimentar no momento certo e de forma gradativa, além de orientar quanto, a saciedade da criança de acordo com sua faixa etária, visando sempre a condição socioeconômica da família. A partir dessas ações será criada uma ponte entre o profissional de enfermagem e as mães, para que seja possível facilitar o aprendizado e alcançar o crescimento e desenvolvimento saudável dessa criança. Por fim, através da ação educativa houve de maneira considerável, a contribuição para um melhor conhecimento da mãe a respeito do assunto exposto.

\section{REFERÊNCIAS}

1. ARAÚJO TS, et al. Desnutrição infantil em um dos municípios de maior risco nutricional do Brasil: estudo de base populacional na Amazônia Ocidental Brasileira. Revista Brasileira de Epidemiologia, 2016; 2016; 19(3): 554-566.

2. CARLETTI CO, et al. Exposição pós-natal a poluentes (MPS 2, 5) como fator de risco para o desenvolvimento como fade comportamentos de depressão e ansiedade: Um estudo experimental. Revista Unoeste, 2019; 3(4): 564-569.

3. CARDOSO AVP, et al. A introdução alimentar em lactentes e aspectos relacionados. Revista Educação em Saúde. Revista Eletrônica Educação em Saúde ,2019; 7(Sup.2): 203-214.

4. GURMINI J, et al. Análise da alimentação complementar em crianças entre 0 a 2 anos de vida de escolas públicas. Revista Medica da UFPR, 2017; 4(2).

5. HANZEN IP, et al. Diagnósticos, intervenções e resultados de enfermagem para a consulta de enfermagem à criança. Enfermagem em Foco, 2019; 10(7): 16-21.

6. LOPES WC, et al. Alimentação de crianças nos primeiros dois anos de vida. Revista Paulista de Pediatria, 2018;36(2): 164-170.

7. MINISTÉRIO DA SAÚDE. Manual de quadros de procedimentos AIDPI Criança: 2 meses a 5 anos. Organização PanAmericana da Saúde Fundo das Nações Unidas para a Infância 2017a. Disponível em: http://bvsms.saude.gov.br/publicacoes/manual_quadros_procedimentos_aidpi_crianca_2meses_5anos.pdf. Acessado em: 6 de janeiro de 2022.

8. MINISTÉRIO DA SAÚDE. Política Nacional de Atenção à Saúde da Criança: orientações para implementação. $2018 \mathrm{~b}$. Disponível em:https://bvsms.saude.gov.br/.iff.fiocruz.br/biblioteca/pnaisc. Acessado em: 6 de janeiro de 2022.

9. MINISTÉRIO DA SAÚDE. Secretaria de Atenção Primaria à Saúde. Departamento de Promoção da Saúde. Guia alimentar para crianças brasileiras menores de 2 anos 2019c Disponível em http://bvsms.saude.gov.br/publicacoes/guia alimentar para crianças brasileiras menores de 2 anos. Acessado em 6 de janeiro de 2022.

10. MORAIS MB, et al. Hábitos e atitudes de mães de lactentes em relação ao aleitamento natural e artificial em 11 cidades brasileiras. Revista Paulista de Pediatria, 2017; 35(1)

11. NARCHI NZ, et al. Variáveis que influenciam a manutenção do aleitamento materno exclusivo. Revista Escola de Enfermagem, 2018; 43(1): 87-94.

12. PEDRAZA DF, OLIVEIRA MM. Estado nutricional de crianças e serviços do saúde prestados por equipes de saúde em família. Ciência e Saúde Coletiva, 2021; 26 (8).

13. RAMOS DC, COELHO TCB. Representação social de mães sobre alimentação e uso de estimulantes do apetite em crianças: satisfação, normalidade e poder. Physis: Revista de Saúde Coletiva, 2017; 27(2).

14. SILVA IE, et al. A importância do enfermeiro no aleitamento materno exclusivo para a evolução da criança. Revista Brasileira Interdisciplinar de Saúde (ReBIS), 2020; 2(1): 7-13.

15. SILVEIRA RSO, et al. Construção de Tecnologia Educativa para Incentivar Puérperas ao Aleitamento Materno. Revista Mostra Interdisciplinar do Curso de Enfermagem, 2016; 2(1). 